\title{
BLOWUP OF SOLUTIONS OF A NONLINEAR WAVE EQUATION
}

\author{
ABBES BENAISSA AND SALIM A. MESSAOUDI
}

Received 4 April 2001 and in revised form 20 October 2001

We establish a blowup result to an initial boundary value problem for the nonlinear wave equation $u_{t t}-M\left(\left\|B^{1 / 2} u\right\|^{2}\right) B u+k u_{t}=|u|^{p-2} u, x \in \Omega$, $t>0$.

\section{Introduction}

We consider the initial boundary value problem (IBVP) for the nonlinear wave equation

$$
\begin{array}{ll}
u_{t t}-A u+k u_{t}=|u|^{p-2} u, & x \in \Omega, t>0, \\
u(x, t)=0, & x \in \partial \Omega, t \geq 0, \\
u(x, 0)=u_{0}(x), \quad u_{t}(x, 0)=u_{1}(x), & x \in \Omega,
\end{array}
$$

where

$$
\begin{gathered}
A u=M\left(\left\|B^{1 / 2} u\right\|^{2}\right) e^{-\Phi(x)} \operatorname{div}\left(e^{\Phi(x)} \nabla u\right), \\
\left\|B^{1 / 2} u\right\|^{2}=\int_{\Omega} e^{\Phi(x)}|\nabla u|^{2} d x,
\end{gathered}
$$

$p>2$ is a constant, $k$ is a positive constant, $M: \mathbb{R}_{+} \rightarrow \mathbb{R}_{+}$is a continuous function, $\Phi \in L^{\infty}(\Omega)$, and $\Omega \subset \mathbb{R}^{n}$ is a bounded domain with a smooth boundary $\Gamma$ so that the divergence theorem can be applied.

When $M \equiv 1$ and $\Phi \equiv 0$, for the case $k=0$, it is well known that the source term $|u|^{p-2} u$ is responsible for finite blowup (global nonexistence) of solutions with negative initial energy (see $[1,9])$. The interaction 
between the damping term and the source has been first considered by Levine $[11,12]$. For $k>0$, the author showed that solutions, with negative initial energy, blow up in finite time. In [5], Georgiev and Todorova extended Levine's result to the case of nonlinear damping of the form $\left|u_{t}\right|^{m} u_{t}$. This result was generalized to an abstract setup by Levine and Serrin [14], Levine et al. [13], and Vitillaro [18]. In [16], Messaoudi extended the result of Levine to the situation where $\Phi \neq 0$.

When $\Phi \equiv 0$ and $M$ is not a constant function, the equation without the damping and source terms is often called the wave equation of Kirchhoff type which has been introduced by Kirchhoff [10] in order to study the nonlinear vibrations of an elastic string. The existence of local and global solutions in Sobolev and Gevrey classes was investigated by many authors (see $[2,3,4,6,7,8,15,17]$ ).

In the present paper, we investigate the blowup of solutions of the initial boundary value problem (1.1). We show that, for suitably chosen initial data, any strong solution blows up in finite time. Our work is based on the results of [14].

\section{Main result}

In order to state our main result, we introduce the weighted space

$$
\begin{gathered}
L^{S}(\Omega, \Phi):=\left\{v: \Omega \longrightarrow \mathbb{R} / \int_{\Omega} e^{\Phi(x)}\left|u_{0}\right|^{s} d x<\infty\right\}, \\
E(0)=\frac{1}{2} \int_{\Omega} e^{\Phi(x)} u_{1}^{2} d x+\frac{1}{2} \bar{M}\left(\left\|B^{1 / 2} u_{0}\right\|^{2}\right)-\frac{1}{p} \int_{\Omega} e^{\Phi(x)}\left|u_{0}\right|^{p} d x .
\end{gathered}
$$

We also make the following hypothesis:

$$
M \in C\left(\mathbb{R}_{+}, \mathbb{R}_{+}\right), \quad \bar{M}(s)=\int_{0}^{s} M(k) d k,
$$

such that

$$
r \bar{M}(s) \geq s M(s), \quad \forall s \geq 0,1<r<\frac{p}{2} .
$$

Theorem 2.1. Let $p>2$ and assume that (2.2) and (2.3) hold. Then, for any initial data satisfying $E(0)<0$, the solution of (1.1) blows up in finite time.

Proof. Except for the operator $A u$, this problem is similar to [14, problem (4.1)-(4.3)] for $l=2$. So the proof goes exactly like the one of [14, Theorem 5]. It remains only to show that $A u$ and $F(u)=|u|^{p-2} u$ satisfy 
conditions (1s) and (2s) in [14, page 346]. To do this, we set

$$
\begin{gathered}
V=Y=L^{2}(\Omega, \Phi), \quad W=L^{p}(\Omega, \Phi), \\
D=H_{0}^{1}(\Omega, \Phi)=\left\{u \in H_{0}^{1}(\Omega, \Phi) / u, \nabla u \in Y\right\} .
\end{gathered}
$$

It is clear that $A$ and $F$ are Frechet derivatives of the $C^{1}$ real-valued potentials given by

$$
\mathcal{A} u=\frac{1}{2} \bar{M}\left(\left\|B^{1 / 2} u\right\|^{2}\right), \quad \mathcal{F}(u)=\frac{1}{p}\|u\|_{W}^{p} .
$$

Now we have, by virtue of (2.3),

$$
\begin{aligned}
&\langle A u, u\rangle_{V}=\int_{\Omega} e^{\Phi(x)} u M\left(\left\|B^{1 / 2} u\right\|^{2}\right) e^{-\Phi(x)} \operatorname{div}\left(e^{\Phi(x)} \nabla u\right) \\
&=M\left(\left\|B^{1 / 2} u\right\|^{2}\right) \int_{\Omega} u \operatorname{div}\left(e^{\Phi(x)} \nabla u\right) \\
&=\left(\left\|B^{1 / 2} u\right\|^{2}\right)\left\|B^{1 / 2} u\right\|^{2} \leq r \bar{M}\left(\left\|B^{1 / 2} u\right\|^{2}\right) \leq 2 r A u, \\
&\langle F(u), u\rangle_{V}-2 r \mp(u)=\left(1-\frac{2 r}{p}\right)\|u\|_{W}^{p}=(p-2 r) \mp(u) .
\end{aligned}
$$

Therefore, conditions (1s) and (2s) in [14, page 346] are satisfied. This completes the proof.

Remark 2.2. Conditions (3s) and (1d)-(3d) of [14] are automatically satisfied since $P u_{t}=u_{t}$ and $Q\left(t, u_{t}\right)=k u_{t}$ are linear. See the proof of [14, Theorem 5].

\section{Acknowledgments}

The authors would like to thank the referee for his valuable remarks. Special thanks from the second author goes to King Fahd University of Petroleum and Minerals Library for its continuous support.

\section{References}

[1] J. M. Ball, Remarks on blow-up and nonexistence theorems for nonlinear evolution equations, Quart. J. Math. Oxford Ser. (2) 28 (1977), no. 112, 473-486.

[2] P. D'Ancona and S. Spagnolo, Global solvability for the degenerate Kirchhoff equation with real analytic data, Invent. Math. 108 (1992), no. 2, 247-262.

[3] R. W. Dickey, Infinite systems of nonlinear oscillation equations related to the string, Proc. Amer. Math. Soc. 23 (1969), 459-468. 
108 Blowup of solutions of a nonlinear wave equation

[4] _ Infinite systems of nonlinear oscillation equations, J. Differential Equations 8 (1970), 16-26.

[5] V. Georgiev and G. Todorova, Existence of a solution of the wave equation with nonlinear damping and source terms, J. Differential Equations 109 (1994), no. 2, 295-308.

[6] D. Gourdin and M. Mechab, Problème de Goursat non linéaire dans les espaces de Gevrey pour les équations de Kirchhoff généralisées, J. Math. Pures Appl. (9) 75 (1996), no. 6, 569-593 (French).

[7] F. Hirosawa, Global solvability for the generalized degenerate Kirchhoff equation with real-analytic data in $\mathbf{R}^{n}$, Tsukuba J. Math. 21 (1997), no. 2, 483-503.

[8] K. Kajitani and K. Yamaguti, On global real analytic solutions of the degenerate Kirchhoff equation, Ann. Scuola Norm. Sup. Pisa Cl. Sci. (4) 21 (1994), no. 2, 279-297.

[9] V. K. Kalantarov and O. A. Ladyzhenskaya, The occurence of collapse for quasilinear equations of parabolic and hyperbolic type, J. Soviet Math. 10 (1978), 53-70.

[10] G. Kirchhoff, Vorlesungen über Mechanik, Teubner, Leipzig, 1883 (German).

[11] H. A. Levine, Instability and nonexistence of global solutions to nonlinear wave equations of the form $P u_{t t}=-A u+\mathcal{F}(u)$, Trans. Amer. Math. Soc. 192 (1974), $1-21$.

[12] - Some additional remarks on the nonexistence of global solutions to nonlinear wave equations, SIAM J. Math. Anal. 5 (1974), 138-146.

[13] H. A. Levine, S. Ro Park, and J. Serrin, Global existence and global nonexistence of solutions of the Cauchy problem for a nonlinearly damped wave equation, J. Math. Anal. Appl. 228 (1998), no. 1, 181-205.

[14] H. A. Levine and J. Serrin, Global nonexistence theorems for quasilinear evolution equations with dissipation, Arch. Rational Mech. Anal. 137 (1997), no. 4, 341-361.

[15] L. A. Medeiros and M. M. Miranda, Solutions for the equation of nonlinear vibrations in Sobolev spaces of fractionary order, Mat. Apl. Comput. 6 (1987), no. 3, 257-276.

[16] S. A. Messaoudi, Blow up in solutions of a semilinear wave equation, Int. J. Appl. Math. 1 (1999), no. 6, 621-626.

[17] S. Spagnolo, The Cauchy problem for Kirchhoff equations, Rend. Sem. Mat. Fis. Milano 62 (1992), 17-51 (1994).

[18] E. Vittilaro, Global nonexistence theorems for a class of evolution equation with dissipation, Arch. Rational Mech. Anal. 137 (1997), 341-361.

Abbes Benaissa: Department of Mathematics, Djillali Liabes University, P.O. Box 89, Sidi Bel Abbes 22000, Algeria

Salim A. Messaoudi: Mathematical Sciences Department, King Fahd University of Petroleum and Minerals, Dhahran 31261, Saudi Arabia

E-mail address: messaoud@kfupm.edu.sa 


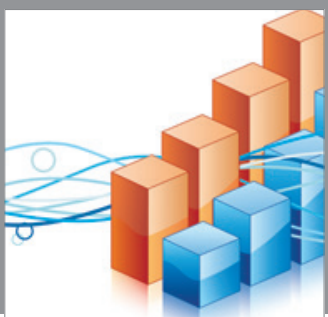

Advances in

Operations Research

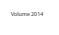

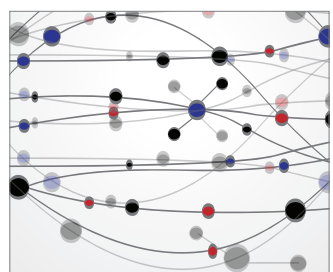

\section{The Scientific} World Journal
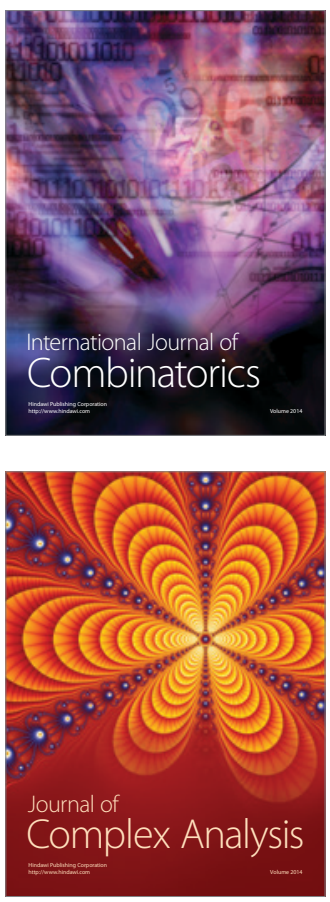

International Journal of

Mathematics and

Mathematical

Sciences
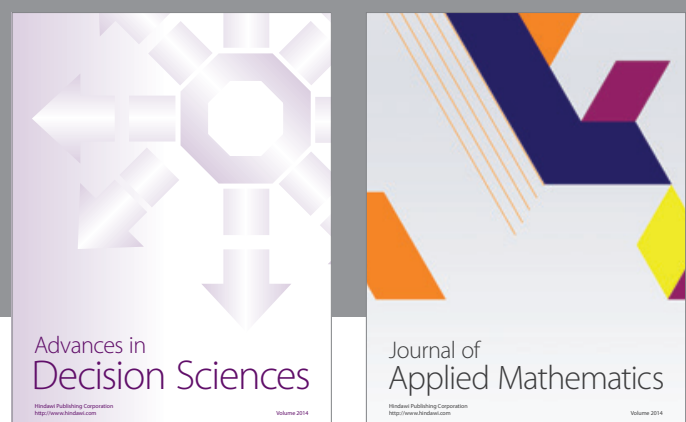

Journal of

Applied Mathematics
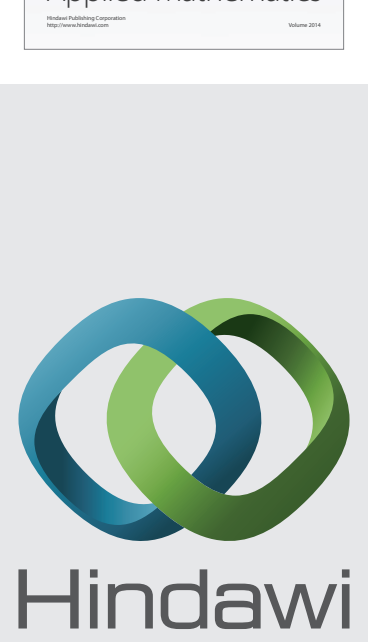

Submit your manuscripts at http://www.hindawi.com
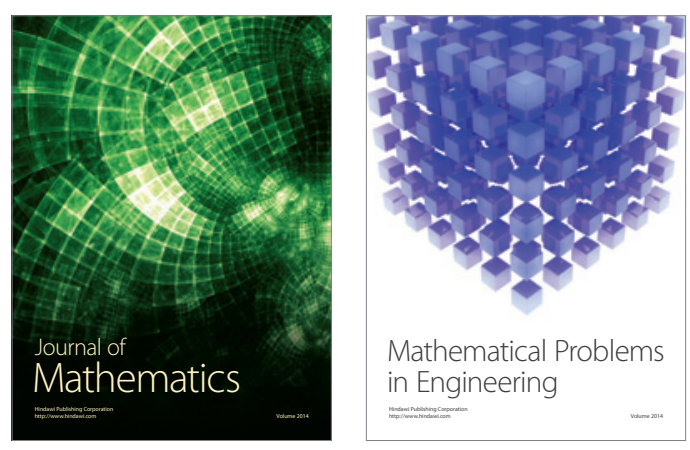

Mathematical Problems in Engineering
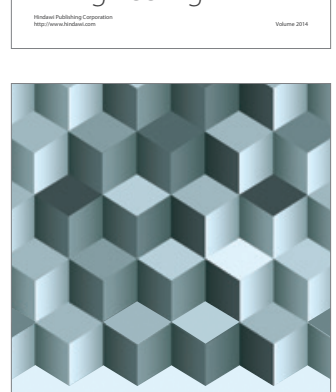

Journal of

Function Spaces
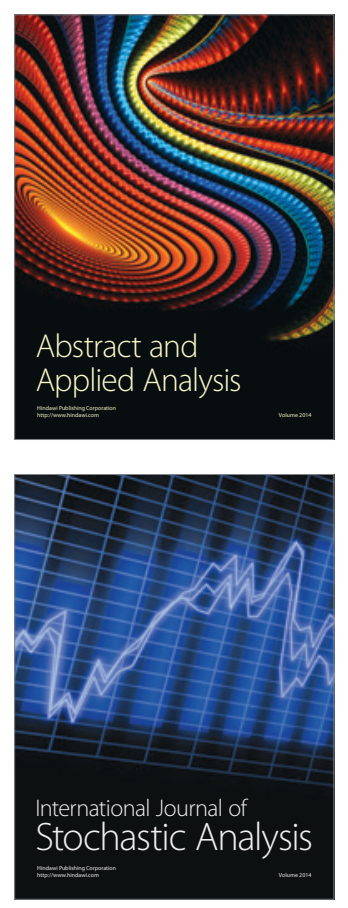

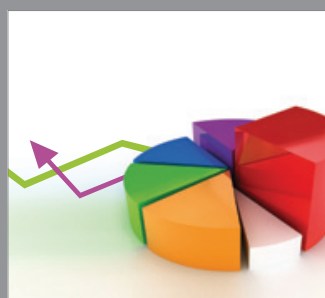

ournal of

Probability and Statistics

Promensencen
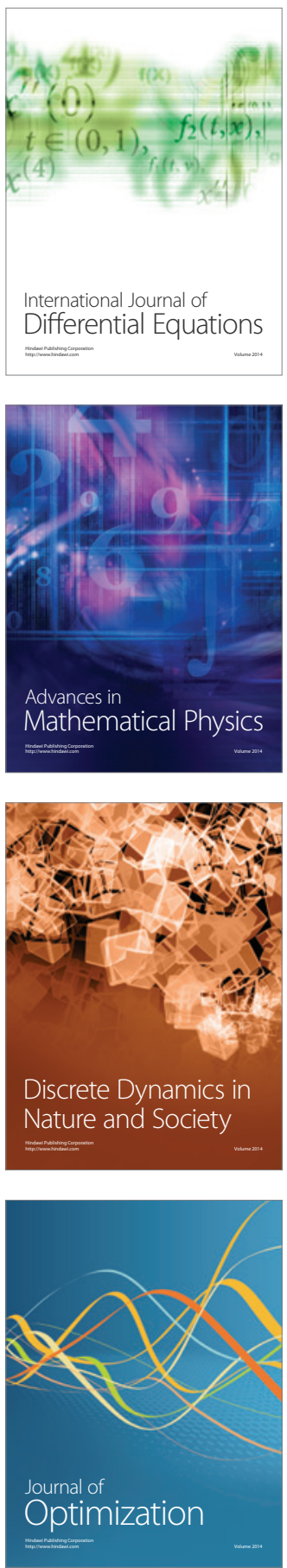